\title{
On the stationary eccentricity of a system of four-tangent conics.
}

\author{
By F. E. Edwardes, B.A.
}

(Received July 1909. Read 12th November 1909).

Let the convex quadrilateral formed by the four given tangents be $A B A^{\prime} B^{\prime}$, and $O$ the intersection of the diagonals. Let $O A$ and $\mathrm{OB}$ be taken as axes of $x$ and $y$. Denote $\mathrm{OA}, \mathrm{OA}^{\prime}, \mathrm{OB}$ and $\mathrm{OB}^{\prime}$ by $a, a^{\prime}, b$ and $b^{\prime}, a$ and $b$ being positive, and $a^{\prime}$ and $b^{\prime}$ negative. The tangential equation of the system is then

$$
(a l+1)\left(a^{\prime} l+1\right)+k(b m+1)\left(b^{\prime} m+1\right)=0,
$$

where $k$ is a variable parameter : for the equation is satisfied when the straight line $l x+m y+1=0$ passes through any two adjacent angular points of the quadrilateral.

The co-ordinates of $\Omega$, the centre of the conic, are

$$
\frac{a+a^{\prime}}{2(1+k)}, \quad \frac{k\left(b+b^{\prime}\right)}{2(1+k)} \text {. }
$$

Hence $\frac{\mathrm{A}_{0} \Omega}{\Omega \mathrm{B}_{0}}=k, \quad \mathrm{~A}_{0}$ and $\mathrm{B}_{0}$ being the middle points of $\mathrm{AA}^{\prime}$ and $\mathrm{BB}^{\prime}$.

Now the Cartesian equation corresponding to the tangential equation

$$
\text { is } \quad \begin{gathered}
a l^{2}+2 h l m+b m^{2}+2 g l+2 f m+c=0 \\
\mathrm{~A} x^{2}+2 \mathrm{H} x y+\mathrm{B} y^{2}+2 \mathrm{G} x+2 \mathrm{~F} y+\mathrm{C}=0,
\end{gathered}
$$

where $\mathrm{A}$, etc., are the co-factors of $a$, etc., in $\triangle$.

The condition for an ellipse is $\mathrm{AB}-\mathrm{H}^{2}>0$, or $c \triangle>0$.

Hence in our case the conic is an ellipse when

$$
-\frac{k(1+k)}{4}\left\{a a^{\prime}\left(b-b^{\prime}\right)^{2} k+b b^{\prime}\left(a-a^{\prime}\right)^{2}\right\}>0 .
$$

Thus $k$ positive gives an ellipse.

If $k$ is negative and numerically less than 1 , i.e. if $\Omega$ lies in the interval $A_{0} \infty$ on the line of centres, the condition for an ellipse is

or

$$
\begin{gathered}
a a^{\prime}\left(b-b^{\prime}\right)^{2} k+b b^{\prime}\left(a-a^{\prime}\right)^{2}>0, \\
1>-k>\frac{b b^{\prime}\left(a-a^{\prime}\right)^{2}}{a a^{\prime}\left(b-b^{\prime}\right)^{2}} .
\end{gathered}
$$


Hence in order that there may be an ellipse with $\Omega$ in $A_{0} \infty$ we must have

$$
\begin{array}{ll} 
& \frac{b b^{\prime}}{\left(b-b^{\prime}\right)^{2}}>\frac{a a^{\prime}}{\left(a-a^{\prime}\right)^{2}}, \\
\text { i.e. } & \frac{\left(b+b^{\prime}\right)^{2}}{\left(b-b^{\prime}\right)^{2}}>\frac{\left(a+a^{\prime}\right)^{2}}{\left(a-a^{\prime}\right)^{2}} \\
\text { i.e. } & \frac{\mathrm{OB}_{0}{ }^{2}}{\mathrm{BB}^{\prime 2}}>\frac{\mathrm{OA}_{0}{ }^{2}}{\mathrm{AA}^{\prime 2}} .
\end{array}
$$

We are at liberty to choose our axes so that this condition is fulfilled.

When $k$ is negative and numerically greater than 1 the condition $\frac{\mathrm{OB}_{0}{ }^{2}}{\mathrm{BB}^{\prime 2}}>\frac{\mathrm{OA}_{0}{ }^{2}}{\mathrm{AA}^{\prime 2}}$ precludes the existence of an ellipse with $\Omega$ in the interval $\mathrm{B}_{0} \infty$.

Further it is easy to show that $k=-\frac{b b^{\prime}\left(a-a^{\prime}\right)^{2}}{a a^{\prime}\left(b-b^{\prime}\right)^{2}}$ when $\Omega$ is at $\mathrm{C}_{0}$, the middle point of the third diagonal of the quadrilateral.

Thus if $\frac{\mathrm{OB}_{0}^{2}}{\mathrm{BB}^{2}}>\frac{\mathrm{OA}_{0}^{2}}{\mathrm{AA}^{2 / 2}}, \mathrm{C}_{0}$ is on $\mathrm{B}_{0} \mathrm{~A}_{0}$ produced and when $\Omega$ is in $\infty \mathrm{C}_{0}$ the conic is an ellipse, ${ }^{*}$

$$
\begin{aligned}
& \text { " " } \quad \mathrm{C}_{0} \mathrm{~A}_{0}, \quad \text { " }, \mathrm{a} \text { hyperbola, } \\
& " \text { " } " \mathrm{~A}_{0} \mathrm{~B}_{0}, ", " \text { an ellipse, }{ }^{*} \\
& \text { " " " } \mathrm{B}_{0 \infty} " \text { " " a hyperbola. }
\end{aligned}
$$

The critical points are $\infty, \mathrm{C}_{0}, \mathrm{~A}_{0}$ and $\mathrm{B}_{0}$, where the values of $k$ are $-1,-\frac{b b^{\prime}\left(a-a^{\prime}\right)^{2}}{a a^{\prime}\left(b-b^{\prime}\right)^{2}}, 0$ and $\infty$. When $\Omega$ passes through $\infty$ the conic changes from an ellipse to a hyperbola through the parabolic form, and when $\Omega$ passes through the other critical points the conic changes from an ellipse to a hyperbola through the linear form. The eccentricity is 1 at each of these points.

- The ellipsea of the system are always real, for the equation referred to the centre ss origin is $A x^{2}+2 H x y+B y^{2}+\frac{\nabla}{A B-H^{2}}=0$, where $\nabla=\left|\begin{array}{lll}A & H & G \\ G & B & F \\ G & F & C\end{array}\right|=\Delta^{2}$. Hence the ellipses are real as long as $B$ is negative. Now $B=k a a^{\prime}-\frac{\left(a-a^{\prime}\right)^{2}}{4}$ which is negative when $k$ is within the elliptio limits. 
The scheme below shows these facts at a glance: $\infty_{\infty} \mathrm{C}_{0} \mathrm{~A}_{0} \mathrm{~B}_{0 \infty}$ is the line of centres, and $\mathrm{C}_{0}$ is situated as represented by virtue of the condition $1>\frac{b b^{\prime}\left(a-a^{\prime}\right)^{2}}{a a^{\prime}\left(b-b^{\prime}\right)^{2}}$.

\begin{tabular}{cccccc} 
ellipse & hyperbola & ellipse & hyperbola \\
\hline$\infty$ & $\mathrm{C}_{0}$ & $\mathbf{A}_{0}$ & $\mathrm{~B}_{0}$ & $\infty$
\end{tabular}

$k=-1 \quad k=-1 \frac{b b^{\prime}\left(a-a^{\prime}\right)^{2}}{a a^{\prime}\left(b-b^{\prime}\right)^{2}} \quad k=0 \quad k=\infty \quad k=-1$

The eccentricity of the system is continuous and has therefore at least four stationary values, a minimum when $\Omega$ is in $\infty \mathrm{C}_{0}$ a maximum when in $\mathrm{C}_{0} \mathrm{~A}_{0}$, a minimum when in $\mathrm{A}_{0} \mathrm{~B}_{0}$ and a maximum when in $\mathrm{B}_{0}$.

We now proceed to find the equation in $k$ which gives the values of the parameter for the conics of stationary eccentricity. The eccentricity is continuous and stationary when $\frac{(\mathrm{A}+\mathrm{B}-2 \mathrm{H} \cos \omega)^{2}}{\mathrm{AB}-\mathrm{H}^{2}}$ is stationary. Straightforward analysis leads to the biquadratic $\mathrm{F}(k) \equiv a a^{\prime}\left(b-b^{\prime}\right)^{4} k^{4}-b b^{\prime}\left(a-a^{\prime}\right)^{4}$

$+2 k\left\{k^{2}\left(b-b^{\prime}\right)^{2}-\left(a-a^{\prime}\right)^{2}\right\}\left\{a a^{\prime}\left(b-b^{\prime}\right)^{2}+b b^{\prime}\left(a-a^{\prime}\right)^{2}\right\}$

$+2 k\left\{a a^{\prime}\left(b-b^{\prime}\right)^{2} k^{2}-b b^{\prime}\left(a-a^{\prime}\right)^{2}\right\}\left\{2\left(a a^{\prime}+b b^{\prime}\right)-\left(a+a^{\prime}\right)\left(b+b^{\prime}\right) \cos \omega\right\}$

$+3 k^{2}\left(b b^{\prime}-a a^{\prime}\right)\left(a-a^{\prime}\right)^{2}\left(b-b^{\prime}\right)^{2}=0$.

The roots of this equation are real and separated by $k=0$, $k=\infty, k=-1$ and $k=-\frac{b b^{\prime}\left(a-a^{\prime}\right)^{2}}{a a^{\prime}\left(b-b^{\prime}\right)^{2}}$.

For $\mathrm{F}(0)$ is positive and $\mathrm{F}(\infty)$ is negative.

Also $\mathbf{F}(-1)=-\left\{\left(a+a^{\prime}\right)^{2}+\left(b+b^{\prime}\right)^{2}-2\left(a+a^{\prime}\right)\left(b+b^{\prime}\right) \cos \omega\right\}$ $\times\left\{a a^{\prime}\left(b-b^{\prime}\right)^{2}-b b^{\prime}\left(a-a^{\prime}\right)^{2}\right\}$,

which is positive by virtue of our supposition $\frac{\mathrm{OB}_{0}{ }^{2}}{\mathbf{B B}^{2}}>\frac{\mathrm{OA}_{0}{ }^{2}}{\mathbf{A A}^{2{ }^{2}}}$.

Further $F\left\{-\frac{b b^{\prime}\left(a-a^{\prime}\right)^{2}}{a a^{\prime}\left(b-b^{\prime}\right)^{2}}\right\}$

$=-\frac{b b^{\prime}\left(a-a^{\prime}\right)^{4}}{a^{3} a^{\prime 3}\left(b-b^{\prime}\right)^{4}}\left\{-a^{2} a^{\prime 2}\left(b-b^{\prime}\right)^{2}-b^{2} b^{\prime 2}\left(a-a^{\prime}\right)^{2}+2 a a^{\prime} b b^{\prime}\left(a+a^{\prime}\right)\left(b+b^{\prime}\right) \cos \omega\right\}$
$\times\left\{a a^{\prime}\left(b-b^{\prime}\right)^{2}-b b^{\prime}\left(a-a^{\prime}\right)^{2}\right\}$,

which is negative.

Hence the biquadratic has four real roots, one of which is positive and three negative. The positive root gives the inscribed ellipse of minimum eccentricity: the negative root between 
-1 and $-\frac{b b^{\prime}\left(a-a^{\prime}\right)^{2}}{a a^{\prime}\left(b-b^{\prime}\right)^{2}}$ the escribed ellipse of minimum eccentricity : and the other negative roots the hyperbolas of maximum eccentricity.

$I$ have not obtained the roots of the quartic in the general case, and doubt whether they could be interpreted geometrically. The geometrical solution of the problem is complicated by the fact that the ranges and pencils involved are not homographic. In certain cases the quartic is less formidable, notably when $\mathrm{ABA}^{\prime} \mathrm{B}^{\prime}$ is cyclic, though why this case lends itself to simplification is not geometrically obvious.

When the quadrilateral is cyclic $a a^{\prime}=b b^{\prime}$ and the quartic reduces to the quadratics

$$
k^{2}\left(b-b^{\prime}\right)^{2}-\left(a-a^{\prime}\right)^{2}=0
$$

and $k^{2}\left(b-b^{\prime}\right)^{2}+2 k\left\{a^{2}+a^{\prime 2}+b^{2}+b^{\prime 2}-\left(a+a^{\prime}\right)\left(b+b^{\prime}\right) \cos \omega\right\}+\left(a-a^{\prime}\right)^{2}=0$.

The positive root is therefore $\frac{a-a^{\prime}}{b-b^{\prime}}$, so that $\frac{\mathrm{A}_{0} \Omega}{\overline{\Omega B_{0}}}=\frac{\mathrm{AA}^{\prime}}{\mathbf{B B}^{\prime}}$; that is to say, the centre of the inscribed ellipse of minimum eccentricity divides the distance between the middle points of the diagonals in the ratio of the diagonals. This value of $k$ makes $\mathrm{A}=\mathrm{B}$, so that the diagonals of the quadrilateral are parallel to equal diameters of the ellipse. The geometrical treatment in this case should be practicable.

When $b=n a, b^{\prime}=n a^{\prime}$ the quadrilateral is a trapezium. $\mathrm{C}_{0}$ is at infinity and the elliptic interval $\mathrm{C}_{0 \infty}$ disappears. One root of the quartic is -1 , and the others are given by the cubic

$$
(k+1)^{2}\left(n^{2} k-1\right)+\frac{\left(a+a^{\prime}\right)^{2}}{\left(a-a^{\prime}\right)^{2}} k(k-1)\left(n^{2}-2 n \cos \omega+1\right)=0 .
$$

If the trapezium is isosceles $n=1$ and $k=1$ is a root, so that the inscribed ellipse of minimum eccentricity is symmetrically placed in the trapezium. The cubic seems no more amenable than the quartic.

If $a=-a^{\prime}$ and $b=-b^{\prime}$ the quadrilateral is a parallelogram, and the $k$ equation becomes

$$
\left(k b^{2}-a^{2}\right)(k+1)^{8}=0 .
$$

The eccentricity of the inscribed ellipse is a minimum when $k=\frac{a^{2}}{b^{2}}$, in which case $\mathrm{H}=0$ and $\mathrm{A}=\mathrm{B}$. The diagonals of the parallelogram are therefore the equi-conjugate diameters of the ellipse. 